\title{
AN ADAPTIVE APPROACH TO TACKLE LOCALIZATION PHENOMENA IN CLUSTERING-BASED REDUCED ORDER MODELS
}

\author{
$\underline{\text { Bernardo P. Ferreira }}^{1,2 *}$, F. M. Andrade Pires ${ }^{1}$ and M. A. Bessa ${ }^{2}$ \\ ${ }^{1}$ Department of Mechanical Engineering, Faculty of Engineering, University of Porto \\ ${ }^{2}$ Faculty of Mechanical, Maritime and Materials Engineering, Delft University of Technology \\ * Corresponding author: bpferreira@fe.up.pt
}

Composites span a broad application spectrum by exploring multiple component synergies into a single material. New manufacturing processes have further widened this spectrum, enabling material microstructure manipulation and creating new design possibilities. Yet, this raises new challenges for computational materials design due to the amount of design possibilities and the complex multi-scale mechanisms that can be explored. Machine learning offers a powerful solution to address such challenges but only if large enough databases can be generated, as highlighted by Bessa and co-workers [1]. Therefore, fast and accurate computational analyses of material representative volume elements (RVEs) are essential because standard direct numerical simulation (DNS) methodologies, such as finite element analyses, are too costly in this context.

In this context, advanced reduced-order models (ROM) have to strike an appropriate balance between accuracy and computational cost. A recent family of clustering-based ROMs [2, 3] is based on the spatial domain decomposition through unsupervised clustering algorithms and has shown promising results in the multi-scale modeling of heterogeneous materials with nonlinear elastoplastic constitutive behavior. However, these methods show clear limitations when localization phenomena take place within RVEs because the domain decomposition lacks the required adaptivity throughout a general deformation path. We propose a novel approach that offers a flexible solution to this significant challenge and demonstrate how clustering-based ROMs, namely the self-consistent clustering analysis (SCA) method [2], can be improved by illustrating its performance for particle reinforced composites. The approach is believed to open important avenues in data-driven materials design.

\section{References}

[1] M.A. Bessa et al. (2017) A framework for data-driven analysis of materials under uncertainty: Countering the curse of dimensionality, Comp Methods Appl M, Vol. 320, pp. 633-667.

[2] Z. Liu, M.A. Bessa and W.K. Liu (2016) Self-consistent clustering analysis: An efficient multi-scale scheme for inelastic heterogeneous materials, Comp Methods Appl M, Vol. 306, pp.319-341.

[3] S. Wulfinghoff, F. Cavaliere and S. Reese (2017) Model order reduction of nonlinear homogenization problems using a Hashin-Shtrikman type finite element method, Comp Methods Appl M, Vol. 330, pp. 149-179. 